\title{
INTERAÇÕES MEDICAMENTOSAS EM PACIENTES CRÔNICOS NA CLÍNICA ODONTOLÓGICA: REVISÃO LITERÁRIA
}

\section{ARTIGO DE REVISÃO}

RODRIGUES, Thayza Cro Alfaro ${ }^{1}$

RODRIGUES, Thayza Cro Alfaro. Interações medicamentosas em pacientes crônicos na clínica odontológica: revisão literária. Revista Científica Multidisciplinar Núcleo do Conhecimento. Ano 06, Ed. 06, Vol. 11, pp. 154-175. Junho de 2021.

ISSN:

2448-0959,

Link

de

acesso: https://www.nucleodoconhecimento.com.br/odontologia/pacientes-cronicos, DOI: 10.32749/nucleodoconhecimento.com.br/odontologia/pacientes-cronicos

\section{RESUMO}

O perfil das doenças no Brasil mudou. Enfermidades infecciosas, como a tuberculose, que eram responsáveis por cerca de $46 \%$ das mortes na década de 90 , hoje foram substituídas por crônicas que, segundo dados do Ministério da Saúde, são responsáveis por $48,9 \%$ dos óbitos. Aliado a este novo cenário, encontramos o crescente envelhecimento da população brasileira e consequente aumento da terceira idade, grupo no qual as moléstias crônicas são altamente prevalentes. Diante da expansão do número de intercorrências advindas da quantidade imensa de fármacos ingeridos diariamente por esses pacientes, é de suma importância que o cirurgiãodentista esteja capacitado para promover, de forma segura, prescrição de medicamentos. Sendo assim, o intuito deste trabalho é realizar a revisão literária dos assuntos vinculados à interação medicamentosa inerentes às alterações metabólicas,

\footnotetext{
${ }^{1}$ Graduação Odontologia pela Universidade Santa Cecília, Pós Graduanda em Saúde Pública, pela Universidade Paulista.
}

RC: 89059

Disponível em: https://www.nucleodoconhecimento.com.br/odontologia/pacientes-cronicos 
de maneira resumida e aplicada, aos quais essa nova gama de pacientes mostra-se exposta.

Palavras-chave: Intercorrência, Interação medicamentosa, Doenças crônicas, Envelhecimento.

\section{INTRODUÇÃO}

O Brasil vem registrando nos últimos anos novo perfil de mortes. De acordo com o Ministério da Saúde (MS), entre 1990 e 2017, houve o acréscimo de 27\% em mortes causadas por doenças crônicas não transmissíveis (DCNT), sendo as doenças cardiovasculares, renais crônicas, respiratórias crônicas obstrutivas, neoplasias e diabetes seus maiores representantes, responsáveis por $72 \%$ dos falecimentos (GARRIDO, 2020).

Fatores econômicos e sociais, tais como a queda da taxa de natalidade, aumento do envelhecimento, redução de doenças ligadas a situações vulneráveis de infraestrutura e baixa escolaridade, somados a fatores de risco modificáveis, como, por exemplo, alimentação inadequada, tabagismo, falta de atividade física, uso abusivo de álcool e obesidade, são elementos preponderantes que levam ao aumento das DCNT, conforme apurado nos Inquéritos de Saúde de Base Populacional realizados pelo IBGE em parceria com o MS.

Além disso, a população brasileira manteve a tendência de envelhecimento nos últimos anos e ganhou 4,8 milhões de idosos desde 2012, superando a marca dos 28 milhões em 2018. Hoje, os idosos representam 13\% da população (IBGE, 2018).

Sendo assim, o aumento da prevalência de doenças crônicas não transmissíveis, unido a majoração da longevidade, torna cada vez mais corriqueiro o uso de múltiplos medicamentos. No Brasil, a Pesquisa Nacional sobre Acesso, Utilização e Promoção do Uso Racional de Medicamentos (PNAUM) estimou em 9,4\% a prevalência do uso

RC: 89059

Disponível em: https://www.nucleodoconhecimento.com.br/odontologia/pacientes-cronicos 
de cinco ou mais remédios entre adultos, também conhecida pelo termo "Polifarmácia".

No entanto, ainda é esperado um aumento progressivo dessa proporção com o envelhecimento populacional, sendo que, entre idosos, essa predominância apresentar-se-á quase duas vezes maior, segundo Projeção da População, divulgada em 2018 pelo IBGE.

Daí porque de ímpar relevância avaliar a real necessidade de novas prescrições de fármacos, e, ainda, o custo-benefício da terapêutica, na hipótese de seu uso se mostrar imprescindível.

Infelizmente, durante a formação do cirurgião-dentista não há grande conscientização dos riscos intrínsecos às interações de medicamentos e da fisiopatologia das doenças crônicas, fatores responsáveis muitas vezes pelo desencadeamento de eventos adversos graves, que, não raro, evoluem para internações hospitalares e até mesmo óbitos. A ideia de que a terapêutica medicamentosa seja coadjuvante ao tratamento proposto (e não o tratamento em si), invoca, portanto, a necessidade de maior enfoque nos meios acadêmicos.

Partindo disso, no auxílio de tornar a prescrição da drogas na rotina odontológica cada vez mais eficaz, nesse artigo serão apresentados, resumidamente: os principais medicamentos utilizados no controle das alterações fisiológicas de pacientes crônicos e idosos; as alterações fisiológicas mais comuns capazes de interferir na farmacocinética; e as possíveis interações adversas aos fármacos mais prescritos em Odontologia para o controle da dor, inflamação, infecção e ansiedade, tornando a farmacovigilância no dia a dia da clínica odontológica algo mais constante.

RC: 89059 


\section{REVISÃO DE LITERATURA}

A prescrição combinada de vários medicamentos a um mesmo paciente é uma prática normal não só no Brasil como em outros países, apesar das recomendações da Organização Mundial da Saúde feitas no sentido de evitá-la.

Em 1985, durante a Conferência Mundial sobre Uso Racional de Medicamentos de Nairobi, definiu-se, inclusive, que "Existe uso racional quando os pacientes recebem medicamentos apropriados a suas necessidades clínicas, em doses adequadas às particularidades individuais, por período de tempo adequado e com baixo custo para eles e sua comunidade".

Nada obstante, o Brasil está entre os 10 maiores mercados farmacêuticos do mundo e representa o principal mercado na América Latina, o que indica seu trilhar em sentido oposto à mencionada diretriz.

O mau uso de remédios deve-se a diversas causas, mas as de grande relevância são a automedicação, multiplicidade de produtos farmacológicos para fins idênticos e a maçante publicidade farmacêutica que instiga o profissional da saúde a prescrever sem conhecimento prévio, atitude que, em boa parte das vezes, resulta no aumento do custo final do tratamento e/ou mesmo provoca intercorrências graves.

Aliada aos pacientes que possuem agravos crônicos, há grande parcela da população que realiza automedicação. Muitos fatores predispõem ao mau hábito, porém a demora nos atendimentos somada às crendices populares, fazem com que a automedicação seja ainda mais frequente e perigosa, sendo negligenciada pelo prescritor que não indaga ao paciente quanto à ingestão de fármacos sem qualquer indicação.

Os medicamentos mais consumidos, sem qualquer prescrição, são os analgésicos e relaxantes musculares, que, pese aparentemente inofensivos, desencadeiam complicações graves, por vezes ocultas, dificultando o diagnóstico.

RC: 89059

Disponível em: https://www.nucleodoconhecimento.com.br/odontologia/pacientes-cronicos 
Entre 2008-2011, foram registrados no Brasil, pelo Sistema Nacional de Informações Tóxico-Farmacológicas, Sinitox, 390.221 casos de intoxicação humana por agentes tóxicos, observando na estatística que em primeiro lugar estão os medicamentos entre os agentes tóxicos, com uma média de $27.55 \%$ dos casos registrados (...) (ROCHA, 2014)

Conforme, ainda, dados colhidos, no ano de 2002, pela Comissão Parlamentar de Inquérito sobre os Medicamentos, $15 \%$ da população consumia, naquela feita, $50 \%$ do que se produzia de remédios, enquanto $51 \%$ entre os com renda de até quatro salários-mínimos consumiam 16\% (ANVISA, 2006).

A interação medicamentosa representa a alteração do efeito terapêutico esperado de determinado fármaco, ocasionado por diversos fatores presentes no momento de sua metabolização. Dentre eles, encontram-se: comorbidades individuais, determinantes genéticos, polifarmácia, alimentos, fitoterápicos e condições farmacodinâmicas inerentes aos medicamentos, como por exemplo, competição por receptores específicos.

Por vezes, o uso associado de medicamentos pode ser benéfico, trazendo resposta terapêutica melhor do que sua utilização isolada; todavia, as interações que mais aqui interessam são as que podem levar a grandes efeitos adversos ao paciente.

Sob esse enfoque, o resultado da utilização concomitante de fármacos para o paciente pode ser classificado como fraco, moderado ou grave. Ao contrário do que ocorre com o moderado, que deve ser acompanhado dada a ligeira possibilidade toxicidade, o fraco possui pouca importância clínica e não deve interferir no planejamento terapêutico. Já na grave, demanda ainda maior atenção dos profissionais, porque resulta em toxicidade crítica exigindo mudança na dose, posologia ou suspensão na sua administração (Tabela 1).

RC: 89059

Disponível em: https://www.nucleodoconhecimento.com.br/odontologia/pacientes-cronicos 
Tabela 1 - Interações medicamentosas

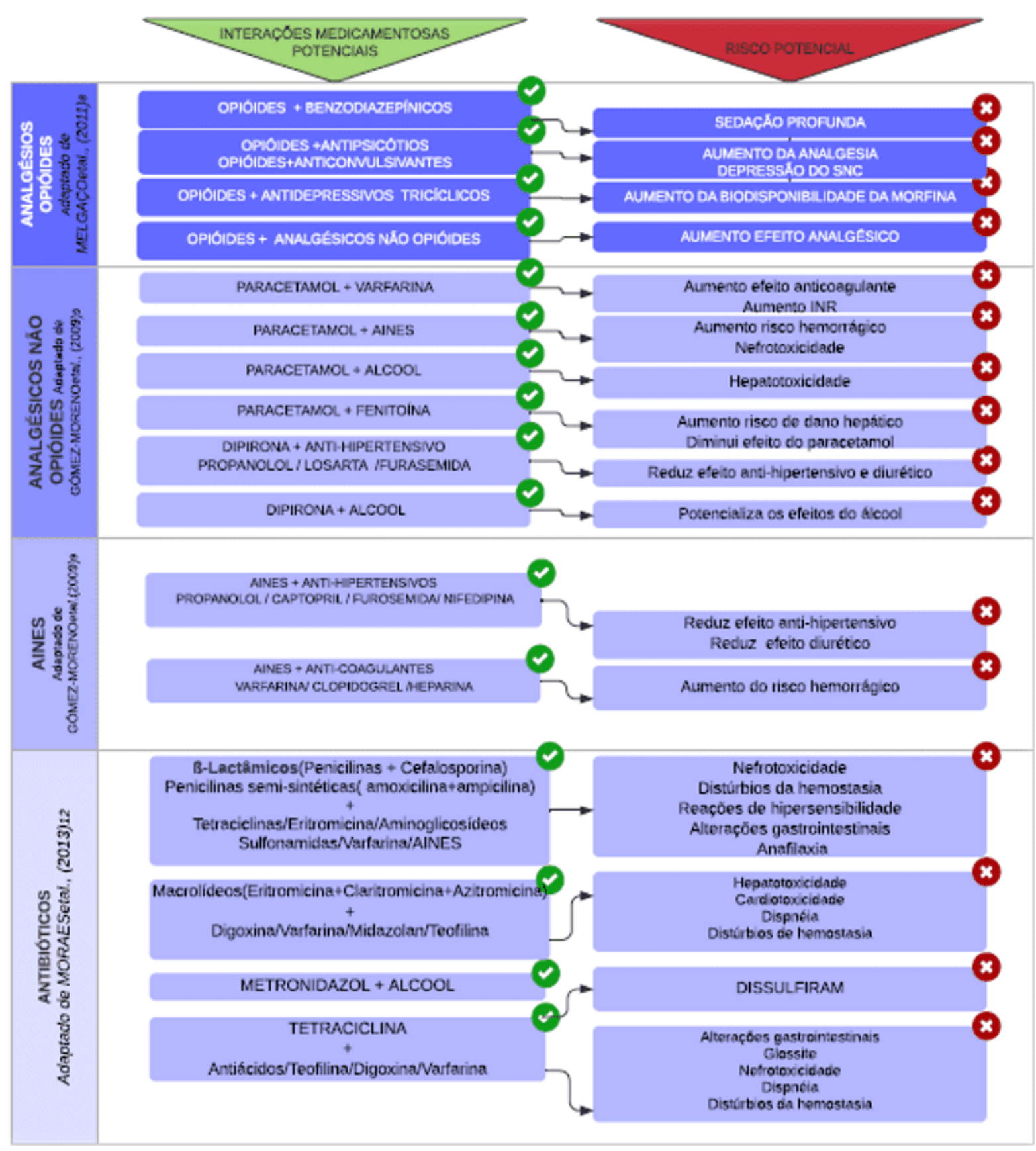

Fonte: Adaptado pela autora.

RC: 89059

Disponível em: https://www.nucleodoconhecimento.com.br/odontologia/pacientes-cronicos 
Além das interações fármaco-fármaco, devemos levar em conta particularidades fisiológicas que estão vinculadas aos pacientes idosos ou portadores de doenças crônicas.

\subsection{IDOSOS}

Entre idosos, a utilização da "polifarmácia" pode provocar efeitos adversos que contribuem para uma sobrecarga funcional grave que ocasionalmente dificulta a recuperação.

O processo de envelhecimento provoca prejuízos às reservas funcionais de vários sistemas, sendo os órgãos mais afetados: rins, fígado, sistema digestório, musculoesquelético, cardiovascular e sistema nervoso central (AIZENSTEIN, 2016).

Com a diminuição da perfusão sanguínea, a velocidade de biotransformação no fígado é reduzida aumentando a meia vida do fármaco, ou seja, o medicamento permanece mais tempo no organismo até ser excretado definitivamente contribuindo para intoxicações.

Em geral, o paracetamol após metabolização hepática transforma-se em substância tóxica para os rins, e sendo a filtração glomerular também reduzida, haverá chances de ocorrer toxicidade sistêmica.

Além disso, devido à diminuição das proteínas plasmáticas responsáveis pelo carreamento das drogas, fármacos como os benzodiazepínicos, anticoagulantes e hipoglicemiantes permanecem em concentrações elevadas no plasma sanguíneo contribuindo para interações graves (Tabela 1).

Dentre os mais velhos, enfermidades como diabetes, hipertensão, problemas cardiorrespiratórios, artrite e câncer são acometimentos comuns e mesmo quando a polifarmácia é realizada de forma consciente haverá o risco de hospitalizações decorrentes de fraturas, pneumonia, hipoglicemia, desnutrição e sangramentos.

RC: 89059

Disponível em: https://www.nucleodoconhecimento.com.br/odontologia/pacientes-cronicos 
Interações com plantas medicinais e álcool também são relatadas com certa incidência entre as pessoas da terceira idade. O uso concomitante de álcool e antiinflamatórios não esteroidais, inclusive o ácido acetilsalicílico (AAS), pode aumentar o tempo de sangramento e predispor à inflamação e hemorragia gástrica.

O acúmulo de acetaldeído provoca reações muito desagradáveis, tais como palpitações, queda da pressão arterial, dor no peito, dificuldade respiratória, vermelhidão da face e pescoço, náuseas, vômitos e transpiração excessiva.

A etanolemia pode aumentar em pacientes utilizando bloqueadores do
receptor H2. O efeito dissulfiram, decorrente do acúmulo de
acetaldeído pela inibição da enzima aldeído desidrogenase, também
representa importante interação álcool-droga, podendo ocorrer
quando são ingeridos medicamentos como o metronidazol, algumas
cefalosporinas e as sulfonilureias. (GOTARDELO, 2015, p. 364).

Pacientes epiléticos com comprometimento cardiovascular e psiquiátrico devem ser avaliados constantemente, uma vez que os antiepiléticos, tal como a fenitoína, reduzem a ação das varfarinas que podem levar a eventos trombóticos de grande relevância.

A varfarina é um anticoagulante oral muito prescrito que age como antagonista da vitamina K. Sua administração concomitante aos antibióticos eritromicina, claritromicina ou metronidazol em tratamentos de 5-8 dias pode levar a um aumento da atividade anticoagulante ocasionando hemorragia.

Em 1992, foi criada uma lista por Beer's et al. (1992) com os principais medicamentos que trazem mais malefícios que benefícios aos idosos; no mesmo ano, a Associação Americana de Geriatria atualizou a relação.

Dentre os medicamentos de uso corrente na odontologia e que causam sérias interação medicamentosas estão os benzodiazepínicos, anti-inflamatórios esteroidais (AIES) e não esteroidais (AINES), e por fim, as aspirinas.

RC: 89059

Disponível em: https://www.nucleodoconhecimento.com.br/odontologia/pacientes-cronicos 
De um modo geral, os benzodiazepínicos causam quedas e amnésia, quando atribuídos a idosos com depressão psíquica. Noutro giro, os AINES, quando associados a pacientes hipertensos, aumentam a retenção de fluidos, ao passo que os AIES podem induzir picos de hiperglicemia em pacientes diabéticos e fraturas em idosos portadores de osteoporose. As aspirinas, a seu turno, quando prescritas a pacientes com problemas crônicos de coagulação podem ocasionar sangramentos e hemorragias graves que levam à hospitalização.

\title{
2.2 HEPATOPATIAS
}

As doenças hepáticas trazem grande preocupação em razão da alta incidência registrada no Brasil. De acordo com a Sociedade Brasileira de Hematologia (SBH), cerca de 30 mil pessoas morrem a cada ano dessas enfermidades, sendo que desse total, a hepatite $C$ afeta $1,5 \%$ da população, ou seja, 2 milhões de pessoas morrem infectadas com o vírus, enquanto a hepatite B restringe-se a 400 mil habitantes.

Em meio a esse cenário, a alteração da função hepática tem consequências marcantes nas concentrações plasmáticas dos fármacos, o que pode reduzir a eficácia e aumentar sua toxicidade.

\begin{abstract}
Determinadas doenças e interações medicamentosas podem afetar a acessibilidade das enzimas hepáticas a agentes farmacológicos. A uremia, causando redução da capacidade de ligação da albumina, facilita a biotransformação de alguns fármacos que se ligam fortemente. (...) O dano hepático pode afetar a apresentação de fármacos ao fígado de diversas formas, podendo acarretar redução da concentração das proteínas plasmáticas e alteração da ligação com fármacos. A diminuição da biotransformação da bilirrubina e de outros substratos também pode alterar a distribuição de um fármaco e sua disponibilidade para captação hepática. Por fim, a cirrose, a insuficiência cardíaca e outras condições que reduzem o fluxo sanguíneo hepático podem retardar de forma significativa a biotransformação (YAGIELA, 2011, p.38)
\end{abstract}

Essas alterações são relevantes quando há necessidade de prescrição medicamentosa. Dos comprometimentos sistêmicos observados em pacientes portadores de cirrose hepática, o sistema nervoso central (SNC) tem sido o mais

RC: 89059

Disponível em: https://www.nucleodoconhecimento.com.br/odontologia/pacientes-cronicos 
afetado, seguido dos efeitos nefrotóxicos que podem ser agravados pelo uso dos aminoglicosídeos (DINIZ, 2010).

Quando presente alterações nas funções renais, distúrbios no sistema reninaangiotensina podem ocorrer. Este sistema é responsável pelo equilíbrio hídrico extracelular e controle da pressão arterial, portanto, sendo neste caso, o uso de AINES deverá ser controlado.

Em geral, o ibuprofeno e o meloxicam são os anti-inflamatórios mais recomendados, seguidos da dexametasona. No entanto, é imprescindível que o médico seja consultado antes de se prescrever o diclofenaco, o cetorolaco ou o celecoxibe (ANDRADE, 2014).

No Brasil ainda não há consenso quanto ao uso da nimesulida, no entanto, vários países da Europa e Estados Unidos suspenderam sua comercialização, após uma série de estudos que vincularam seu uso à ocorrência de hepatotoxicidade.

Dentre os fármacos psicoativos, portadores de insuficiência hepática devem evitar o uso de benzodiazepínicos; todavia, quando imprescindível, o lorazepam pode ser uma opção mais viável com dose reduzida pela metade (DINIZ, 2010).

Apesar de a maioria dos benzodiazepínicos empregados na clínica odontológica ser considerada segura, há razões para crer que a exposição do paciente a vários fármacos com potencial para induzir a porfiria é mais danosa do que a exposição a um só medicamento. Por esse motivo, a sedação só deve ser considerada quando for imprescindível (ANDRADE, 2014, p. 216).

O paracetamol (acetaminofeno), medicamento muito utilizado no Brasil para alívio da dor e febre, deve ter seu uso contido para portadores de insuficiência hepática.

Trata-se do fármaco mais autoadministrado, seguido da dipirona, quando ingerido em doses terapêuticas corretas é considerado seguro; entretanto, seu uso constante e incorreto, pode incidir em lesões hepáticas agudas, ou, como ocorre na maior parte

RC: 89059

Disponível em: https://www.nucleodoconhecimento.com.br/odontologia/pacientes-cronicos 
das vezes, em lesões crônicas e assintomáticas que desencadeiam em câncer de fígado com a necessidade ulterior de transplante (Tabela 1).

Havendo prescrição do metronidazol para estes pacientes crônicos, a real necessidade deverá avaliada. Dentre seus efeitos colaterais, estão: leucopenia, neuropatia, ataxia, tonturas e convulsões. Como já citado, pacientes portadores de insuficiência hepática, em geral, desenvolvem encefalopatias graves e, portanto, o metronidazol pode agravar este quadro. Além disso, podem potencializar quadros de hemorragia, devido a depressão da função hepática, exigindo do profissional acompanhamento constante do paciente, através de mensuração periódica do tempo de protrombina.

O tratamento de infecções fúngicas bucais, por sua vez, exige maior atenção, pois os antifúngicos mais empregados na clínica odontológica (cetoconazol, fluconazol e itraconazol) são classificados como de uso duvidoso (ANDRADE, 2014).

Caso a situação exija profilaxia e tratamento quimioterápico de infecções, as penicilinas devem ser a primeira escolha, por outro lado, pacientes alérgicos devem ser medicados com azitromicina ou doxiciclina. A eritromicina é contraindicada de forma absoluta devido a sua ação hepatóxica já reconhecida.

\subsection{RENAIS}

Os custos gerados para tratamento das doenças renais crônicas (DCR), em especial com as Terapias Renais Substitutivas (TRS) - diálise e/ou transplante renal, são os maiores entre as enfermidades crônicas e consomem valores desproporcionais dos orçamentos destinados à saúde em todos os países. Só em 2014, para se ter uma ideia, foram gastos 2,62 bilhões de reais para tratamento dos DCR no Brasil. (GARRIDO, 2020)

A doença renal afeta mais de 60 milhões de pessoas no mundo. No Brasil, estima-se que mais de 90 mil pessoas sofram de DRC e são dependentes de diálise ou transplantes. Entre elas, 35\% têm idade superior a 65 anos. A cada ano, cerca de 30 mil brasileiros precisam

RC: 89059

Disponível em: https://www.nucleodoconhecimento.com.br/odontologia/pacientes-cronicos 
iniciar tratamento por hemodiálise ou diálise peritoneal e, segundo a Sociedade Brasileira e Nefrologia, mais de 30 mil pacientes se encontram na fila aguardando transplante renal (AIZENSTEIN, p.250, 2016).

$\mathrm{Na}$ insuficiência renal crônica (IRC), há perda das funções renais em decorrência de distúrbios vasculares, glomérulos, túbulos e no interstício renal e no trato urinário inferior, de ordem aguda ou crônica, sendo as principais causas desencadeantes a hipertensão arterial, glomerulonefrite e diabetes.

Estima-se que o número de pessoas portadoras de doença renal crônica seja muito maior do que estimativas oficiais, devido, em grande parte, pela dificuldade em se obter seu diagnóstico precoce. Em geral, os primeiros sinais clínicos da insuficiência renal começam a se manifestar quando $75 \%$ dos néfrons já se encontram deteriorados.

O processo da perda da função renal é caracterizado por estágios clínicos distintos. O primeiro é conhecido como reserva renal diminuída onde há uma perda de 40 a $75 \%$ da função do néfron, e em geral, o paciente não apresenta sintomas porque os néfrons remanescentes são capazes de realizar as funções normais do rim. $\mathrm{O}$ segundo estágio é conhecido como Insuficiência Renal, onde 75 a $90 \%$ da função do néfron foram perdidos (BERREDO, 2003 apud DIAS, 2011).

A redução da função renal provoca retenção de inúmeras substâncias provenientes do metabolismo proteico, alteração da pressão arterial causado pelo aumento do volume plasmático e do hematócrito, além de desequilíbrios nos níveis de potássio, sódio, água e equilíbrio ácido-base (AIZENSTEIN, 2016). A menor produção de eritropoietina no plasma provoca a diminuição das células vermelhas, levando ao quadro de anemia e menor agregação plaquetária aumentando o tempo de sangramento.

A uremia também leva à supressão da resposta linfocitária, disfunção dos granulócitos e resposta imunológica mediada por células. Sendo assim, esses pacientes têm maior predisposição a infecções, sendo a causa mais comum de óbito (ANDRADE, 2014).

RC: 89059

Disponível em: https://www.nucleodoconhecimento.com.br/odontologia/pacientes-cronicos 
O manejo clínico odontológico do paciente portador de DRC, especialmente quando há a necessidade de realizar procedimentos invasivos, deve ser criterioso dada a quantidade de alterações metabólicas associadas à doença.

Pacientes que fazem hemodiálise recebem heparina e por esse motivo devem sempre ser atendidos no dia seguinte ao procedimento. A heparina é um anticoagulante que permite o sangue passar pelo equipamento de diálise sem coagular, e por possuir meia-vida plasmática curta (2-4h), é recomendado que os procedimentos odontológicos que possam causar sangramento sejam agendados para o dia seguinte às sessões de diálise, ante o risco elevado de hemorragia (Tabela 1).

Quando prescrições medicamentosas forem necessárias, além da necessidade de se optar por drogas de metabolização hepática, deve-se considerar o estágio da DRC, taxa de filtração glomerular (TFG) e mecanismo de excreção do medicamento (Figura 1)

Figura 1 - Taxa De Filtração Glomerular (TFG)

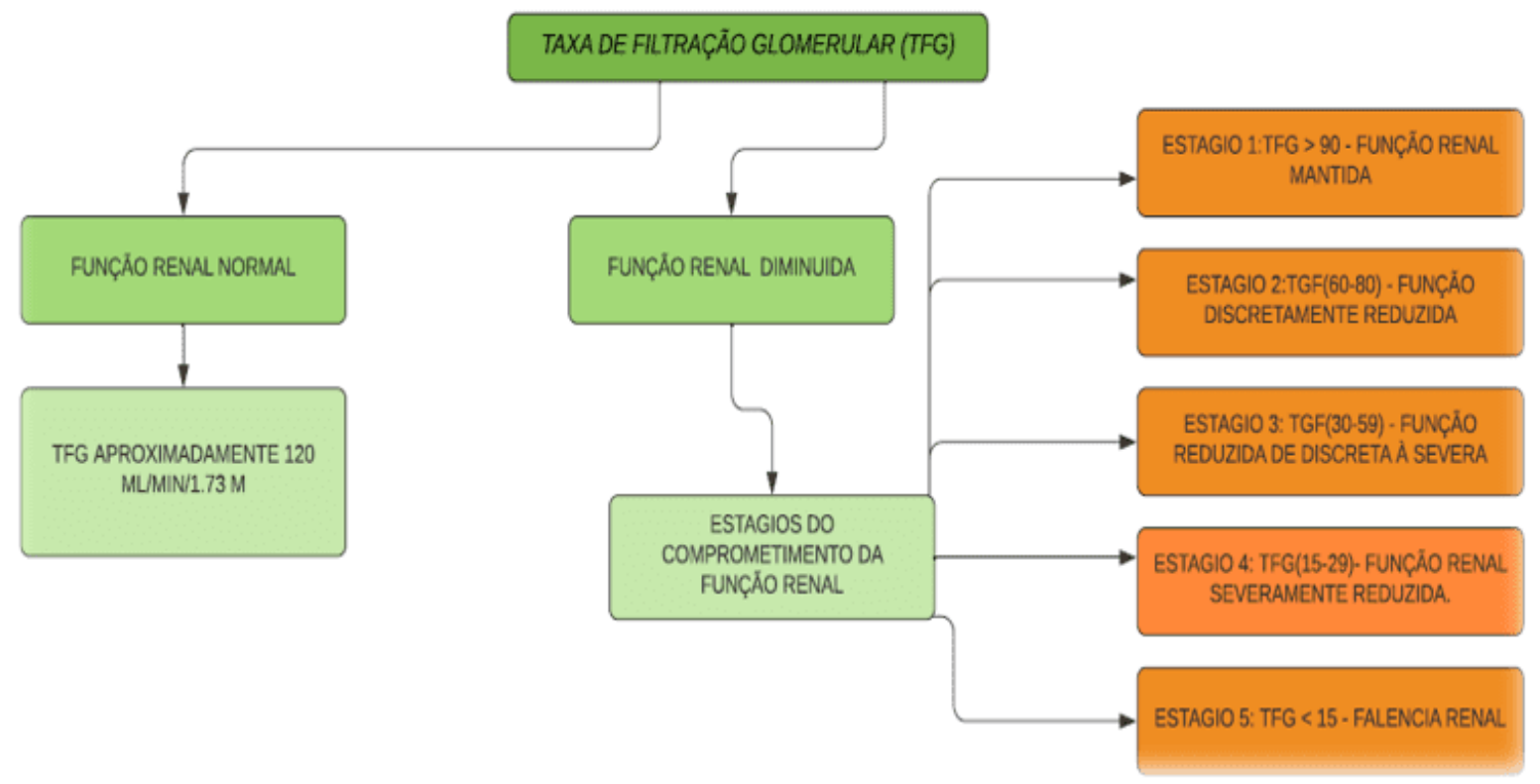

Fonte: Adaptado (GARRIDO, 2020).

RC: 89059

Disponível em: https://www.nucleodoconhecimento.com.br/odontologia/pacientes-cronicos 
Quanto à utilização de analgésicos para dores leves, dipirona e paracetamol, nas menores doses eficazes, podem ser prescritos; o ácido acetilsalicílico, por outro lado, deve ter seu contido, por aumentar o risco de hemorragias. Quadros hemorrágicos são comuns em pacientes renais crônicos devido à toxicidade urêmica que leva a deficiência do Fator III, dificultando a interação entre plaquetas e endotélio. (FILHO, 2006)

(...)os pacientes com DRC apresentarem maior incidência de micro hemorragias cerebrais,infartos cerebrais silenciosos e lesões da substância branca (leucariose), mesmo quando ajustados para fatores de risco comuns (por exemplo, hipertensão e diabetes mellitus). (FRANCO, 2019)

Para dores moderadas a intensas, analgésicos de ação central, tal como o Tramadol, podem ser uma boa escolha.

Nos casos leves da DRC, não há necessidade de redução das doses ou mudanças no intervalo entre as doses dos antibióticos, porém as tetraciclinas e as cefalosporinas devem ser evitadas devido ao seu potencial nefrotóxico.

Pacientes com disfunções renais podem limitar a excreção dos agentes antimicrobianos. $\mathrm{O}$ ajuste das doses dos antibióticos que se excretam pela urina pode realizar-se por meio do aumento dos intervalos entre as doses terapêuticas usuais ou através da variação das doses. Amoxicilina, eritromicina, clindamicina e o metronidazol podem ser usados com precaução. As tetraciclinicas e os aminoglicosídeos estão contraindicados na presença de doença renal (...) uma vez que a vancomicina não pode ser eliminada por métodos dialíticos convencionais, é droga de grande aplicação e comodidade para pacientes em terapêutica substitutiva da função renal por diálise.(FILHO, 2007)

O uso dos AINES também deve ser coibido ao máximo, por aumentar a retenção de sódio e interagir com os anti-hipertensivos e diuréticos, levando ao aumento brusco da pressão arterial e interferência na agregação plaquetária. Os corticosteroides (dexametasona ou betametasona), em dose única ou por tempo restrito, são a melhor escolha para tratamento.

RC: 89059

Disponível em: https://www.nucleodoconhecimento.com.br/odontologia/pacientes-cronicos 


\subsection{HIPERTENSOS}

A hipertensão arterial sistêmica (HAS) é uma condição clínica multifatorial caracterizada por níveis elevados e sustentados de pressão arterial (PA). (SOCIEDADE BRASILEIRA DE CARDIOLOGIA, 2010)

A hipertensão arterial é a DCNT mais prevalente na população masculina até os 50 anos de idade. A partir dessa faixa etária, esses números começam a se inverter, mostrando uma prevalência maior em mulheres. Porém, em ambos os sexos, os números são crescentes com o avançar da idade (GARRIDO, 2020, p.3).

Muitas pessoas são hipertensas e desconhecem sua condição. Sendo assim, recomenda-se de que a pressão arterial deva ser aferida na primeira consulta, mesmo que não haja histórico de HAS (ANDRADE, 2014) (Figura 2).

Figura 2 - Pressão arterial (PA) em maiores de 18 anos

\section{PRESSÃO ARTERIAL(PA) EM}

MAIORES DE 18 ANOS

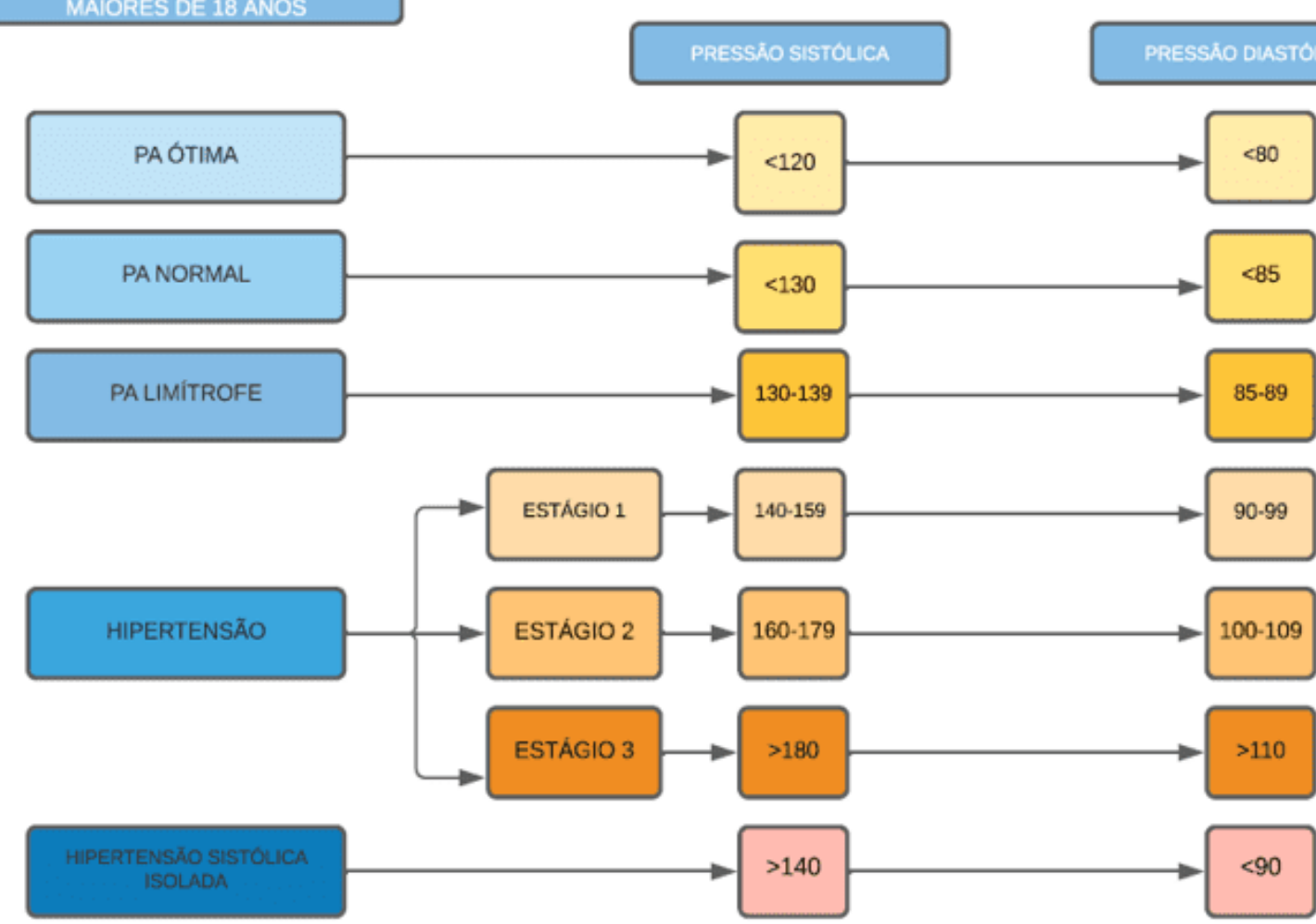

Fonte: Adaptado (GARRIDO, 2020).

RC: 89059

Disponível em: https://www.nucleodoconhecimento.com.br/odontologia/pacientes-cronicos 
O paciente hipertenso diagnosticado e controlado apresenta diversas especificidades, dentre elas uso de medicamentos para o controle da pressão arterial, comorbidades e prejuízos às órgãos-alvos. Muitos desses fármacos levam ao aparecimento de alterações bucais visíveis, sendo necessário o conhecimento por parte do cirurgião dentista ao realizar procedimentos e até mesmo orientar preventivamente o paciente (Figura 3).

Figura 3- Grupo de medicamentos x alterações orais

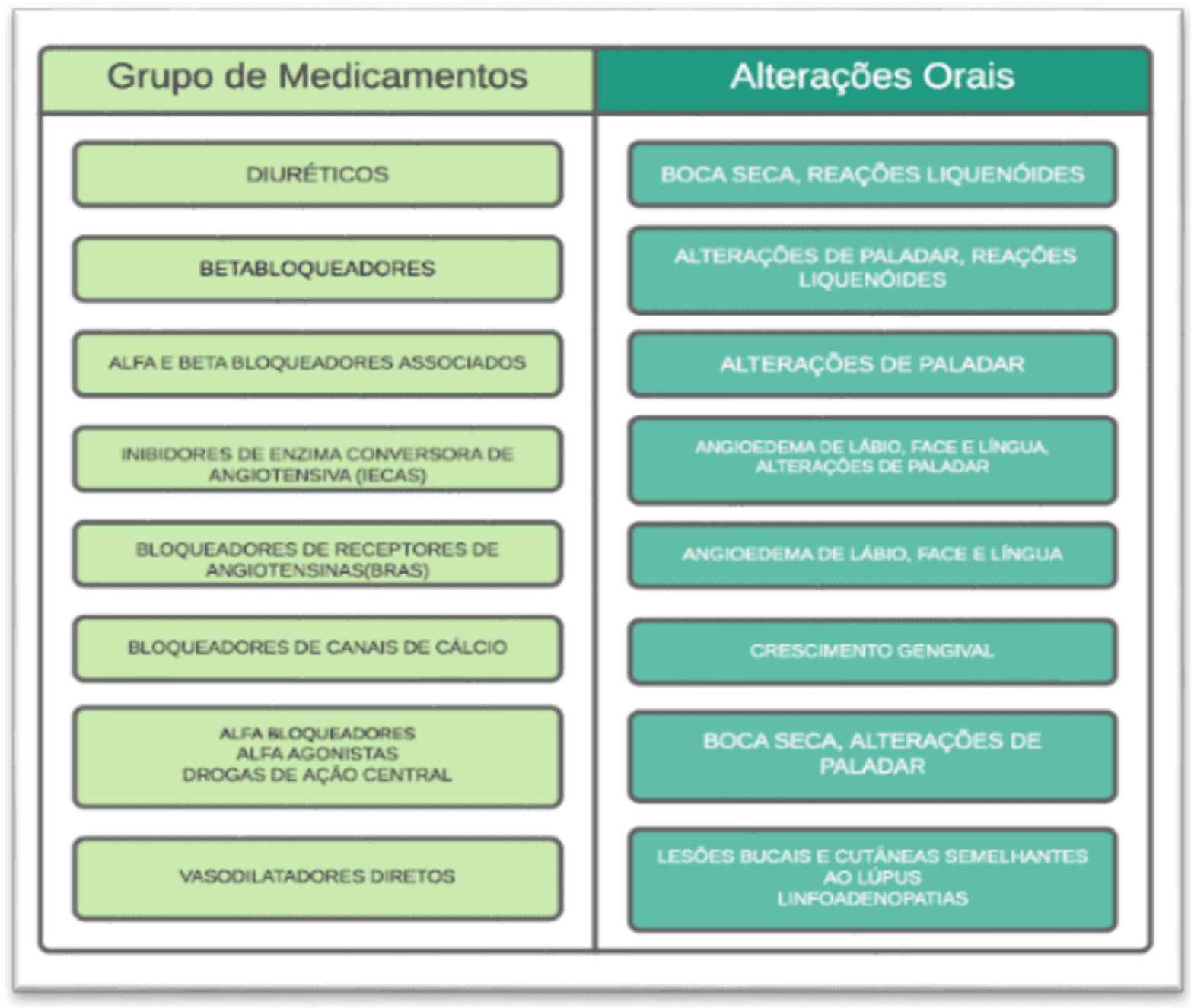

Fonte: Adaptado GARRIDO, 2020.

RC: 89059

Disponível em: https://www.nucleodoconhecimento.com.br/odontologia/pacientes-cronicos 
Baseado neste e em outros critérios, a terapêutica medicamentosa precisa ser criteriosa ao considerar potenciais interações medicamentosas graves que podem acometer tais indivíduos (Tabela 1).

$\mathrm{Na}$ rotina odontológica é muito comum a prescrição de AINES, no entanto, tais fármacos afetam o mecanismo de ação de drogas anti-hipertensivas, devendo, portanto, sua utilização não exceder quatro dias.

Devido a tendência de reduzirem o fluxo sanguíneo nos rins, os AINES diminuem a eficácia de vários medicamentos utilizados para controle da HAS porém dependentes da atividade renal, dentre eles: inibidores da enzima conversora da angiotensina (benazepril, captopril, enalapril, lisinopril); antagonistas $\beta$ Adrenérgicos (atenolol, metoprolol, propranolol); diuréticos de alça (bumetanida,furosemida); diuréticos(hidroclorotiazida); e receptores antagonistas de angiotensina II (candesartan, eprosartana, iresartana, losartana).

Estudos mostraram que a pressão arterial pode aumentar em até $5 \mathrm{mmHg}$, principalmente quando os AINES naproxeno e ibuprofeno são combinados.

Para pacientes hipertensos, o controle de ansiedade é essencial, portanto, benzodiazepínicos são medicamentos muito importantes no manejo odontológicos destes pacientes. Para tanto, recomenda-se a utilização de midazolam, antes dos procedimentos no intuito de evitar aumento súbito da pressão arterial decorrente de questões emocionais.

\subsection{DIABÉTICOS}

No ano de 1980, a Organização Mundial da Saúde (OMS) previu que o número de pessoas diabéticas no mundo seria de 422 milhões em 2014. A prevalência a nível global entre adultos saltou de 4,7\%, em 1980, para 8,5\%, em 2014. Somente no Brasil, existem 14.3 milhões de portadores de diabetes, sendo projetado para 2030 a $6^{a}$ posição do país entre os dez com a maior quantidade de diabéticos.

$\mathrm{RC}: 89059$

Disponível em: https://www.nucleodoconhecimento.com.br/odontologia/pacientes-cronicos 
A diabetes mellitus (DM) é um problema de saúde pública de grande morbidade e mortalidade causadas por suas complicações agudas e crônicas, com altos números de hospitalizações. Trata-se de uma moléstia metabólica sistêmica crônica associada a deficiência parcial ou total de insulina, que acarreta inadequada utilização dos carboidratos e alterações no metabolismo lipídico e proteico.

Subdivide-se em tipo 1, tipo 2, gestacional e outros tipos associados a quadros específicos, dentre eles: DM associado a síndromes genéticas; DM por uso crônico de drogas; DM associado a endocrinopatias ou DM associado à disfunção exócrina do pâncreas.

A DM do tipo 1 ocorre em decorrência da degeneração decorrente de processo autoimune das células beta do pâncreas, afetando, principalmente, crianças e adultos jovens. Por esse motivo, os diabéticos, do Tipo 1, são chamados de insulinodependente, visto que deixam de produzir insulina em totalidade.

Já a DM do tipo 2, em regra, acomete indivíduos obesos com mais de 40 anos de idade, embora hodiernamente se vislumbre aumento de incidência de casos entre crianças e jovens em função de maus hábitos alimentares, sedentarismo e estresse.

O grande problema atrelado ao diabetes tipo 2 é que, em muitas vezes, é assintomática para a maior parte da população. Presume-se que $50 \%$ dos diabéticos não sabem que são portadores da enfermidade permanecendo sem diagnóstico até o surgimento das primeiras intercorrências. Em alguns episódios, podem ser observados os "sintomas clássicos" da doença, também conhecidos como os "4 P's": poliúria, polipepsia, polifagia e perda de peso. Por isso, o conhecimento dos limites de normalidades em exames laboratoriais é importante para garantir a segurança da terapêutica que for ser instituída (Figura 4).

RC: 89059

Disponível em: https://www.nucleodoconhecimento.com.br/odontologia/pacientes-cronicos 
Figura 4- Testes laboratoriais para diagnósticos da diabetes mellitus (DM)

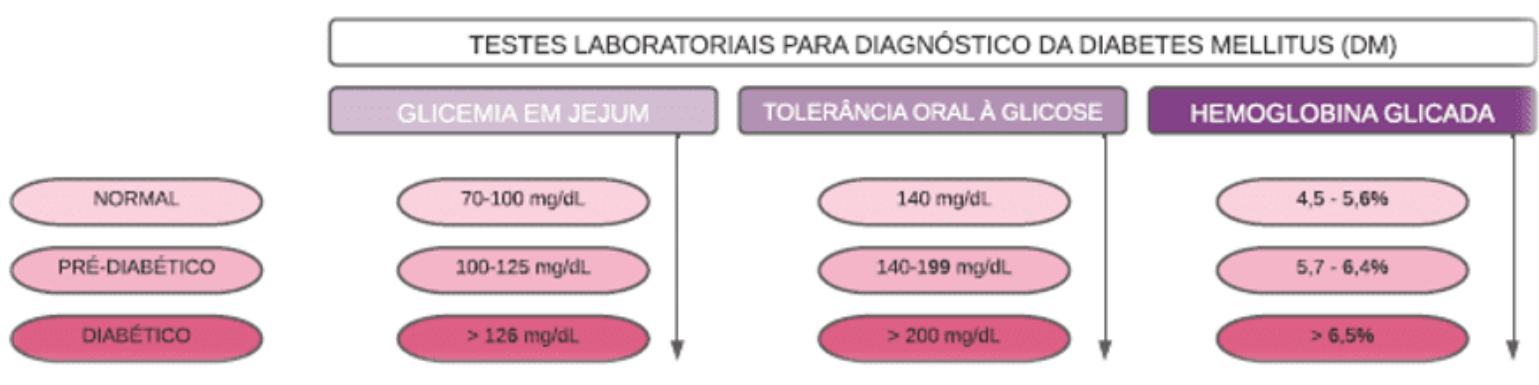

Fonte: Adaptado GARRIDO, 2020.

As pessoas diabéticas sofrem complicações tanto por hiperglicemia quanto por hipoglicemia, sendo as complicações agudas decorrentes das oscilações drásticas de glicose circulante. A hipoglicemia é definida por valores sanguíneos de glicose abaixo de $70 \mathrm{mg} / \mathrm{dl}$ no sangue.

Os medicamentos mais empregados para controle da DM são os hipoglicemiantes orais, sendo as sulfonilureias (clorpropamida e glibenclamida) a primeira escolha para diabéticos de Tipo 2 não obesos e a metformina para tratar diabéticos obesos ou com sobrepeso.

Quando não se obtém resposta à terapia com hipoglicemiantes orais, a insulina pode substituí-los ou a eles se agregar. Outros hipoglicemiantes orais, como inibidores da alfa-glicosidase, metiglinidas e glitazonas, são agentes coadjuvantes instituídos quando a monoterapia não é suficiente para controle da DM (Tabela 1).

A insulina, prescrita principalmente para diabéticos do Tipo 1, pode ser obtida por meios naturais quando extraída do pâncreas de bois e/ou porcos ou por síntese bacteriana (insulina humana). No que diz respeito ao tempo de duração, pode ser de ação curta (rápida), intermediária (lenta) ou prolongada (ultralenta).

Para pacientes controlados, o uso de benzodiazepínico deve ser considerado para prevenção de hiperglicemia decorrente de estresse. Os mais indicados são:

$\mathrm{RC}: 89059$

Disponível em: https://www.nucleodoconhecimento.com.br/odontologia/pacientes-cronicos 
midazolam, alprazolam, diazepam ou lorazepam (idosos), nas mesmas dosagens empregadas em pacientes hígidos. (ANDRADE, 2014).

Em procedimentos mais invasivos, que possam desencadear dor de maior intensidade seguido de edema, uma a duas doses de corticosteroides (dexametasona, betametasona) podem ser utilizadas com segurança. No entanto, os AINES devem ser evitados.

\footnotetext{
A ação hipoglicêmica das sulfonilureias pode ser potencializada por fármacos que apresentam alto grau de ligação proteica, como alguns dos anti-inflamatórios não esteroides (AINEs). Isso significa que os AINEs podem competir com os hipoglicemiantes orais pelos mesmos sítios de ligação às proteínas plasmáticas, deslocando-os e deixandoos na forma livre, o que poderá desencadear um quadro de hipoglicemia. (ANDRADE, 2014)
}

Outra condição que deve ser observada, é que a insuficiência renal crônica. Trata-se de comorbidade presente em $40 \%$ dos pacientes com DM. Portanto, a prescrição à longo prazo de AINES deve ser evitada, devido ao risco de comprometimento irreversível do metabolismo renal.

\section{DISCUSSÃO}

Ainda há muito o que se discutir quanto à prescrição medicamentosa para pacientes crônicos, dada as infinitas possibilidades de intercorrências que podem ocorrer. Retomando à exposição inicial, no Brasil as DCNT correspondem à $72 \%$ do número de enfermos, sendo que $45 \%$ da população adulta, ou seja, 54 milhões de indivíduos relatam ao menos uma doença crônica.

Além da inoperância de políticas públicas de prevenção contra esses agravados, a falta de educação em saúde leva à ausência do autocuidado, impedindo a reflexão pessoal no que se refere à necessidade de consumir tantos medicamentos e a inércia de comportamento que poderia prover melhor qualidade de vida, desvinculando a ideia de que a cura virá somente através de remédios.

RC: 89059

Disponível em: https://www.nucleodoconhecimento.com.br/odontologia/pacientes-cronicos 
Malgrado os esforços legais em mensurar o nível de propagandas que partem da indústria farmacêutica, a compra de drogas sem prescrição ainda é alta, e pior, sem o próprio paciente reconhecer a gravidade que esse tipo de aquisição pode render a si.

No Brasil, entre os anos 2008-2011, foram registrados no SINITOX nada menos do que 390.221 ocorrências de intoxicação humana por agentes tóxicos, sendo o pódio dos agentes causadores encabeçado pelos medicamentos, com $27.55 \%$ de casos (ROCHA, 2014).

Um ponto de partida para que mudança sejam consolidadas é o melhor preparo acadêmico dos profissionais prescritores, com constantes atualizações e estudos, sobretudo, conhecimento dos mecanismos de ação das dezenas de medicamentos que chegam todos os anos ao mercado.

Atualmente, a dinâmica do processo de prescrição tornou-se mais tecnológica, contando com ferramentas que auxiliam no conhecimento das possíveis intercorrências medicamentosas, promovendo desta forma, qualidade e segurança do atendimento.

Uma das ferramentas que pode ser utilizada pelos profissionais de saúde é o sistema de consulta on-line Uptodate $\AA$, que pode ser utilizado para verificar a ocorrência de interações medicamentosas em uma prescrição. Essa é composta por uma base importante de referências bibliográficas e foi projetada para prover informações atualizadas sobre interações medicamentosas. Existem também alguns aplicativos para smartphones como o Medscape e o Micromedex. Esses aplicativos verificam rapidamente as interações medicamentosas de acordo com as necessidades do profissional, de maneira rápida e fidedigna com a literatura. Esses aplicativos podem ser instalados também em tablets, o que pode proporcionar uma prescrição com mais segurança durante uma consulta (BERTOLO, 2013).

Mesmo que a automedicação ainda seja um evento de difícil controle, a prescrição incorreta ou desnecessária é ainda mais grave, pois parte-se do princípio de que o cirurgião dentista esteja habilitado a prescrever os fármacos mais adequados às necessidades do paciente.

RC: 89059

Disponível em: https://www.nucleodoconhecimento.com.br/odontologia/pacientes-cronicos 
Sucede, infelizmente, que a Farmacologia não é a disciplina mais discutida e, por isso, cada vez mais intercorrências se vislumbram.

\section{CONCLUSÃO}

O conhecimento das doenças crônicas não transmissíveis, bem como suas alterações fisiológicas, precisa ser de domínio do cirurgião dentista para que as prescrições farmacológicas sejam seguras.

A farmacodinâmica inerente aos principais medicamentos prescritos na rotina odontológica, também devem ser conhecidos, no intuito de se praticar a farmacovigilância de modo constante.

No entanto, o conhecimento farmacológico nunca deve ser isolado das demais ferramentas clínicas. Realizar anamnese de forma correta é fator primordial, apesar de muitas vezes, negligenciada. Através de um simples questionário, o dentista passa a conhecer o paciente que está sendo atendido.

O tratamento multidisciplinar também precisa ser melhorado. Muitas vezes, por não compreender a gravidade da doença do paciente, o contato com o médico responsável é descartado.

A relação entre o cirurgião-dentista e o médico é de suma importância, visto que ambos podem contribuir para o entendimento do risco/benefício ao se medicar um paciente crônico ou idoso, prevenindo interações farmacológicas iatrogênicas.

Todos os anos, milhares de pessoas são internadas, morrem ou se acidentam, em decorrência de interações medicamentosas que poderiam ter sido evitadas, caso, fossem antevistas. Portanto, espera-se que no futuro, as medicações sejam melhor indicadas em benefício das próximas gerações.

RC: 89059

Disponível em: https://www.nucleodoconhecimento.com.br/odontologia/pacientes-cronicos 


\section{REFERÊNCIAS}

AIZENSTEIN, Moacir Luiz. Fundamentos para o uso racional de medicamentos 3. ed.- Rio de Janeiro: Elsevier, 2016.

ANDRADE, Eduardo Dias. Terapêutica medicamentosa em Odontologia - 3. ed. São Paulo: Artes Médicas, 2014.

ANVISA - AGENCIA NACIONAL DE VIGILANCIA SANITÁRIA. Parcerias para diminuir o mau uso de medicamentos. Revista de Saúde Pública. São Paulo, v.40 n.1, p. 191-194, 2006. Disponível em:< https://scielosp.org/pdf/rsp/2006.v40n1/191192/pt >. Acesso em 31 mar. 2021.

Berrêdo VCM, Carvalho CS. Qualidade de vida de pacientes transplantados renais do Hospital Universitário Unidade Presidente Dutra (HU-UPD) [Trabalho de conclusão de curso]. São Luís (MA): Universidade Federal do Maranhão; 2003.

BERTOLLO, Avner Luis; DEMARTINI, Cristiano; PIATO, Angelo Luis. Interações medicamentosas na clínica odontológica - Drug interactions in dental clinic. Revista Brasileira de Odontologia, Rio de Janeiro, v.70, n. 2, jul.-dez. 2013. Disponível em: < http://revodonto.bvsalud.org/scielo.php?script=sci_arttext\&pid=S003472722013000200005 > . Acesso em 30 mar. 2021;

DIAS, Bruna Neres Dias; CAMARGO, Deizielli Cosma. O perfil do paciente renal crônico no Brasil a partir da prevalência de pacientes em tratamento dial. Monografia (Graduação). Faculdades Integradas Aparício Carvalho - FIMCA. Porto Velho, 2011.

DINIZ, Margareth de Fátima Melo et al. Principais drogas com as possíveis interações medicamentosas prescritas na clínica odontológica. Revista Brasileira de Ciências da Saúde, João Pessoa, Paraíba, v. 13, n. 1, p. 66-70, fev. 2010. Disponível em:< https://periodicos.ufpb.br/ojs/index.php/rbcs/article/view/3707/3639 >. Acesso em 30 mar. 2021;

RC: 89059

Disponível em: https://www.nucleodoconhecimento.com.br/odontologia/pacientes-cronicos 
FIGUEIREDO, Renata Rodrigues. Uso racional de medicamentos na Odontologia: conhecimento, percepções e práticas. 2009. 106. Dissertação (Mestrado) Universidade Federal da Bahia, Salvador, 2009;

FILHO, José Zenou Costa, PADILHA, Walter Suruagy Motta, SANTOS Ellen Karla Nobre. Cuidados odontológicos em portadores de insuficiência renal crônica. Rev. Cir. Traumatol. Buco-Maxilo-fac., Camaragibe v.7, n.2, p. 19 - 28, abr./jun. 2007

FRANCO, Álvaro de Oliveira, STAROSTA, Rodrigo Tzovenos, CRUZ,Matheus Roriz. O impacto específico de toxinas urêmicas em domínios cognitivos: uma revisão. J. Bras. Nefrol. v.41, n.1, p:103-111, 2019.

GARRIDO, D; HADDAD, A. E. Cuidados odontológicos de pacientes com doença renal crônica. In: UNA-SUS/UFMA. Atendimento odontológico em pacientes com Doenças Crônicas Não Transmissíveis. Assistência odontológica a pacientes com DCNT: diabetes, hipertensão e doença renal crônica. São Luís: UNA-SUS/UFMA, 2020.

GÓMEZ-MORENO, Gerardo et al. Pharmacological interactions of anti-inflammatoryanalgesics in odontology. Med Oral Patol Oral Cir Bucal; Valencia, Espanha, v. 14, n. 2, p. 81-89, fev. 2009. Disponível em: < http://www.medicinaoral.com/pubmed/medoralv14_i2_pE81.pdf >. Acesso em 30 mar. 2021;

GOTARDELO, Daniel Riani et al. Consumo de álcool e interações álcool-drogas entre idosos atendidos na Estratégia Saúde da Família -Revista Médica de Minas Gerais, Belo Horizonte, Minas Gerais, v. 25, n. 3, p. 363-368, Jul/Set 2015. Disponível em:< http://www.dx.doi.org/10.5935/2238-3182.20150071 >. Acesso em 30 mar 2021;

IBGE - Retratos, a revista do IBGE. Rio de Janeiro, n. 16, fev.2019. Disponível em: $<$ https://agenciadenoticias.ibge.gov.br/media/com_mediaibge/arquivos/d4581e6bc87 ad8768073f974c0a1102b.pdf > . Acesso em 30 mar 2021;

RC: 89059

Disponível em: https://www.nucleodoconhecimento.com.br/odontologia/pacientes-cronicos 
IORIS, Lisiane Marcelli Dalmédico; Bacchi, André Demambre . Interações medicamentosas de interesse em Odontologia. Revista Da Faculdade De Odontologia - UPF, Passo Fundo, Rio Grande do Sul, v. 24, n. 1, p.148-154, fev 2019. Disponível em:< https://doi.org/10.5335/rfo.v24i1.8807 >. Acesso em 30 mar 2021;

MALTA, Deborah Carvalho et al. Probabilidade de morte prematura por doenças crônicas não transmissíveis, Brasil e regiões, projeções para 2025 - Revista Brasileira de Epidemiologia, São Paulo, v. 22, n. 01, abr. 2019. Disponível em: < https://scielosp.org/article/rbepid/2019.v22/e190030/\# > . Acesso em 30 mar 2021;

MELGAÇO, Tainah Brasil et al. Polifarmácia e ocorrências de possíveis interações medicamentosas. Revista Paraense de Medicina, Belém, Pará, v. 25, n.1, jan.- mar. 2011. Disponível em:< http://files.bvs.br/upload/S/0101-5907/2011/v25n1/a2585.pdf >. Acesso em 30 mar. 2021;

OLIVEIRA, Marcelo Lehnen Rodrigues . Frequência e Caracterização de Potenciais Interações entre medicamentos de uso contínuo com aqueles de uso corrente na prática odontológica. 2018. Dissertação (Trabalho de Conclusão de Curso). Universidade Federal do Rio Grande do Sul, Porto Alegre, 2018;

PADOIN, Karina.; COMARELLA, Larissa; SOLDA, Caroline Medicamentos comumente prescritos na odontologia e suas principais interações medicamentosas: revisão de literatura. Journal of Oral Investigations ; Passo Fundo - RS - Brasil, v. 7, n. 1, p. 62-76, jan.-jun., 2018. Disponível em: < https://pesquisa.bvsalud.org/portal//resource/pt/biblio-915478 >. Acesso em 30 mar. 2021;

ROCHA, Ana Leda Ribeiro. Uso Racional de Medicamentos. 2014. Dissertação (Especialização). FIOCRUZ, Rio de Janeiro, 2014;

RC: 89059

Disponível em: https://www.nucleodoconhecimento.com.br/odontologia/pacientes-cronicos 
SOCIEDADE BRASILEIRA DE CARDIOLOGIA / Sociedade Brasileira de Hipertensão / Sociedade Brasileira de Nefrologia. VI Diretrizes Brasileiras de Hipertensão. Arquivo Brasileiro de Cardiologia 2010; 95(1 supl.1): 1-51

SÍRIO LIBANÊS, Hospital. Doenças crônicas não transmissíveis são maior causa de morte no Brasil. Disponível em: < https://www.hospitalsiriolibanes.org.br/imprensa/noticias/Paginas/Doencas-cronicasnao-transmissiveis-sao-maior-causa-de-morte-no-

Brasil.aspx\#: :text=De\%20acordo\%20com\%20dados\%20oficiais,causas\%20de\%20 morte\%20no\%20Pa\%C3\%ADs. >. Acesso em 30 mar. 2021;

TAVARES, Maria de Souza; MACEDO, Thiago Campelo, MENDES Daniella Ribeiro Guimarães. Possíveis Interações Medicamentosas em um Grupo de Hipertenso e Diabético da Estratégia Saúde da Família. Revista de Divulgação Científica Sena Aires, Valparaíso de Goiás, Goiás, v.1, n.2, p. 119-125, jul. -dez. 2012. Disponível em: < http://revistafacesa.senaaires.com.br/index.php/revisa/article/view/21 >. Acesso em 30 mar. 2021;

WANNMACHER, Lenita; FERREIRA, Maria Beatriz Cardoso. Farmacologia clínica para dentistas - 3. ed. - Rio de Janeiro: Guanabara Koogan, 2007.

YAGIELA, John A. et al. Farmacologia e terapêutica para dentistas - Rio de Janeiro: Elsevier, 2011.

Enviado: Abril, 2021.

Aprovado: Junho, 2021.

RC: 89059

Disponível em: https://www.nucleodoconhecimento.com.br/odontologia/pacientes-cronicos 